\title{
Preconditioners for state constrained optimal control problems with Moreau-Yosida penalty function
}

\author{
John W. Pearson ${ }^{2}$, Martin Stoll ${ }^{1, *}$, Andrew J. Wathen ${ }^{2}$ \\ ${ }^{1}$ Computational Methods in Systems and Control Theory, Max Planck Institute for Dynamics of Complex Technical \\ Systems, Sandtorstr. 1, 39106 Magdeburg Germany, (martin.stoll80@gmail.com) \\ 2 Numerical Analysis Group, Mathematical Institute, 24-29 St Giles', Oxford, OX1 3LB, United Kingdom \\ (wathen@maths.ox.ac.uk)
}

\section{SUMMARY}

Optimal control problems with partial differential equations as constraints play an important role in many applications. The inclusion of bound constraints for the state variable poses a significant challenge for optimization methods. Our focus here is on the incorporation of the constraints via the Moreau-Yosida regularization technique. This method has been studied recently and has proven to be advantageous compared to other approaches. In this paper we develop robust preconditioners for the efficient solution of the Newton steps associated with solving the Moreau-Yosida regularized problem. Numerical results illustrate the efficiency of our approach. Copyright (c) 2011 John Wiley \& Sons, Ltd.

Received ...

KEY WORDS: State constrained problems, PDE-constrained optimization, Saddle point systems, Preconditioning, Newton method, Krylov subspace solver

\section{INTRODUCTION}

Optimization problems with constraints given by partial differential equations (PDEs) arise in a variety of applications (see [24]). Comprehensive introductions to this field can be found in [48, 24]. Throughout this paper, we consider the minimization of a functional $J(y, u)$ defined as

$$
J(y, u):=\frac{1}{2}\left\|y-y_{d}\right\|_{L^{2}(\Omega)}^{2}+\frac{\beta}{2}\|u\|_{L^{2}(\Omega)}^{2},
$$

with $\Omega \subset \mathbb{R}^{\bar{d}}, \bar{d} \in\{2,3\}$. In (1) $\beta \in \mathbb{R}^{+}$represents a regularization parameter and $y_{d}$ is a given function that represents the desired state. The state $y$ and the control $u$ are linked via the Poisson equation

$$
-\triangle y=u \text { in } \Omega
$$

with boundary conditions $y=g$ on $\partial \Omega$ or

$$
-\triangle y+y=u \text { in } \Omega
$$

with boundary conditions $\frac{\partial y}{\partial n}=0$ on $\partial \Omega$. We decide to consider both (2) and (3) as both play a significant role in the literature. The choice of $g$ will typically be 0 or the projection of $y_{d}$ onto the

\footnotetext{
${ }^{*}$ Correspondence to: Computational Methods in Systems and Control Theory, Max Planck Institute for Dynamics of Complex Technical Systems, Sandtorstr. 1, 39106 Magdeburg Germany, (martin.stoll80@ gmail.com)
} 
box defined by constraints. The introduction of box constraints on the control and the state, i.e.,

$$
\underline{u} \leq u \leq \bar{u}
$$

and

$$
\underline{y} \leq y \leq \bar{y}
$$

is of practical interest. In this paper we will focus on the numerical solution of the optimization problem given above when state constraints are present. Effective preconditioning strategies for the control constrained case can be found in [46, 23]. We will later show that a semi-smooth Newton method applied to the Moreau-Yosida regularization of (1) leads to a linear system of saddle point form. The saddle point matrix is symmetric and indefinite, and a variety of methods exists to solve problems of this type efficiently (see [2] for a survey). In practice the linear system is usually of sufficiently high dimension that iterative solution methods are needed, and it is never solved without the application of a preconditioner $\mathcal{P}$ which is chosen to enhance the convergence behaviour of the iterative method. A variety of preconditioners exists to tackle saddle point problems. The aim of this paper is to present preconditioners that are tailored towards the efficient solution of the linear system arising from the discretization of an optimal control problem involving a partial differential equation and state constraints. In general, the state constrained problem is a considerably harder problem (see Section 2) than the control constrained problem. In this paper, we will introduce preconditioning strategies that allow for a robust solution of the linear system with respect to both the regularization parameter $\beta$ and the parameter coming from the Moreau-Yosida penalty term.

The paper is organized as follows. The problem we are interested in will be presented in detail in Section 2. Our focus in this paper is to derive efficient preconditioners for the optimal control problems and hence our focus is to introduce all methods from a linear algebra perspective. We show how for each method the saddle point system can be preconditioned and efficiently solved using a Krylov subspace technique. We successively introduce three preconditioners, where the first is derived from previous results for PDE-constrained optimization and the second follows a recent techniques focusing on robustness with respect to the regularization parameters. The numerical results presented in Section 4 illustrate the performance of the presented method.

\section{THE MOREAU-YOSIDA FORMULATION}

We consider the case when state constraints are introduced and assume that the functional $J(y, u)$ (1) has to be minimized for functions $y$ and $u$ defined over a domain $\Omega \subset \mathbb{R}^{\bar{d}}$. The problem of minimizing (1) when bound constraints on the state are given is more complicated than the control constrained case $[8,24,26]$ as in general the Lagrange multiplier is only a measure. Several remedies have been proposed for this problem. In [30] Meyer et al. consider regularized state constraints , i.e.,

$$
\underline{y} \leq \varepsilon u+y \leq \bar{y}
$$

An alternative approach is given by changing the objective function (1) using the Moreau-Yosida penalty function [25] to give

$$
\begin{aligned}
J(y, u):= & \frac{1}{2}\left\|y-y_{d}\right\|_{L^{2}(\Omega)}^{2}+\frac{\beta}{2}\|u\|_{L^{2}(\Omega)}^{2}+\frac{1}{2 \varepsilon}\|\max \{0, y-\bar{y}\}\|_{L^{2}(\Omega)}^{2} \\
& +\frac{1}{2 \varepsilon}\|\min \{0, y-\underline{y}\}\|_{L^{2}(\Omega)}^{2},
\end{aligned}
$$

subject to the above state equations with appropriate boundary conditions. For the remainder of this manuscript we will assume that the state equations and hence $J(y, u)$ are considered in discretized form using an appropriate finite element discretization [23]. 
The discretized version of state equation (2) and (1) is given by

$$
\begin{aligned}
\text { Minimize } & \frac{1}{2}\left(y-y_{d}\right)^{T} M\left(y-y_{d}\right)+\frac{\beta}{2} u^{T} M u \\
& +\frac{1}{2 \varepsilon} \max \{0, y-\bar{y}\}^{T} M \max \{0, y-\bar{y}\} \\
& +\frac{1}{2 \varepsilon} \min \{0, y-\bar{y}\}^{T} M \min \{0, y-\bar{y}\}
\end{aligned}
$$

subject to $K y=M u-f$.

Here $K$ represents the stiffness matrix and $M$ the mass matrix. We will only consider the lumped mass matrix here but comment later on how to precondition for the consistent mass matrix. Note that $y, u, y_{d}, \bar{y}$, and $\underline{y}$ now represent vectors. The optimality system of (8) looks as follows:

$$
\begin{aligned}
& -K^{T} \lambda=-M\left(y-y_{d}\right)-\varepsilon^{-1} \chi_{\mathcal{A}_{+}} M \max \{0, y-\bar{y}\}-\varepsilon^{-1} \chi_{\mathcal{A}_{-}} M \min \{0, y-\underline{y}\} \\
& \beta M u+M \lambda=0 \\
& -K y+M u=f
\end{aligned}
$$

with $\chi_{\mathcal{A}_{+}}$being the characteristic function for the indices where $y-\bar{y}>0$ and $\chi_{\mathcal{A}_{-}}$the characteristic function for the region where $y-y<0$. Note that $\mathcal{A}_{+}=\left\{i: y_{i}>\bar{y}_{i}\right\}$ and $\mathcal{A}_{-}=$ $\left\{i: y_{i}<\underline{y}_{i}\right\}$ are the active sets associated with the bound constraints on the state $y$ at step $k$. If we now wish to apply a semi-smooth Newton method to (9)-(11) we must solve the following system at every step:

$$
\left[\begin{array}{ccc}
M+\varepsilon^{-1} G_{\mathcal{A}} M G_{\mathcal{A}} & 0 & -K^{T} \\
0 & \beta M & M \\
-K & M & 0
\end{array}\right]\left[\begin{array}{l}
y^{(k+1)} \\
u^{(k+1)} \\
\lambda^{(k+1)}
\end{array}\right]=\left[\begin{array}{c}
c_{\mathcal{A}} \\
0 \\
f
\end{array}\right]
$$

where $c_{\mathcal{A}}=M y_{d}+\varepsilon^{-1}\left(G_{\mathcal{A}_{+}} M G_{\mathcal{A}_{+}} \bar{y}+G_{\mathcal{A}_{-}} M G_{\mathcal{A}_{-}} \underline{y}\right)$ defines part of the right hand side, $\mathcal{A}=$ $\mathcal{A}_{-} \cup \mathcal{A}_{+}$and the $G$ matrices are projections onto the active sets defined by $\mathcal{A}$. The application of the semi-smooth Newton method to these problems has been studied (see [23, 25, 5]). Our task is the efficient solution of the linear system in (12) which is of saddle point form. Note that we do not focus on the discussion of the the inexact semi-smooth Newton method here but rather refer to [27] where it was observed that with suitable preconditioning this method performed just as well as the exact semi-smooth Newton method.

In the case of the state equation being defined by (3) we define $K:=K_{N}+M$, where $K_{N}$ is the stiffness matrix for a pure Neumann problem, and obtain the same formulation as shown above.

The Moreau-Yosida regularization has also recently been analyzed for semilinear elliptic problems (see [28]).

\section{SOLUTION OF THE LINEAR SYSTEM AND EIGENVALUE ANALYSIS}

The system matrix

$$
\mathcal{K}:=\left[\begin{array}{ccc}
L & 0 & -K^{T} \\
0 & \beta M & M \\
-K & M & 0
\end{array}\right]
$$

is symmetric and indefinite; we define $L=M+\varepsilon^{-1} G_{\mathcal{A}} M G_{\mathcal{A}}$ for the remainder of this paper. Note that the block $\operatorname{blkdiag}(L, \beta M)$ is symmetric and positive definite, as we have a mass matrix as the first term and a mass matrix plus a submatrix of a mass matrix as the other. The matrix $K$ is the stiffness matrix associated with the weak formulation of (2) or (3) - it is symmetric and positive definite. Benzi et al. [2] discuss properties and numerical methods to solve matrices of saddle point form. As $\mathcal{K}$ is a large and sparse, symmetric and indefinite matrix, a Krylov subspace 
solver [39, 20, 13] will be our method of choice. For smaller (and typically $2 D$ ) examples direct methods $[9,11]$ will prove very efficient but for large and/or $3 D$ problems these methods are likely to run out of memory.

The choice of preconditioners that we mention in this section is motivated by an observation about the eigenvalues of the preconditioned system $\mathcal{P}^{-1} \mathcal{K}$ for certain preconditioners $\mathcal{P}$. Murphy $e t$ al. show in [31] that for some idealized preconditioners the matrix $\mathcal{P}^{-1} \mathcal{K}$ has only a small number of eigenvalues ( 3 for block-diagonal and 2 for block-triangular).

One method that is a standard choice for symmetric and indefinite systems is the minimal residual method (MINRES) introduced in [32] as a method for minimizing the residual $\left\|r_{k}\right\|_{2}=\left\|\mathcal{K} x_{k}-b\right\|_{2}$ over the current Krylov subspace

$$
\operatorname{span}\left\{r_{0}, \mathcal{K} r_{0}, \mathcal{K}^{2} r_{0}, \ldots, \mathcal{K}^{k-1} r_{0}\right\}
$$

In order to be able to use MINRES, we need the preconditioner to be symmetric and positive definite, and hence block-diagonal preconditioners would present a natural choice [13, 15]. A preconditioner for MINRES and the above problem could look like the following:

$$
\mathcal{P}=\left[\begin{array}{ccc}
A_{0} & 0 & 0 \\
0 & A_{1} & 0 \\
0 & 0 & \widehat{S}
\end{array}\right]
$$

with $A_{0}, A_{1}$, and $\widehat{S}$ being approximations to the $(1,1)$-block, the $(2,2)$-block and the Schur complement, respectively. The use of MINRES for optimal control problems has been recently investigated in [36, 35, 6, 45, 42]. Note that MINRES is also applicable in the case of a semi-definite $(1,1)$-block, which is the case if we were to consider the minimization of $J(y, u)$ as in (1), but with the $\left\|y-y_{d}\right\|^{2}$ term given on some subdomain $\Omega_{1} \subset \Omega$ (as opposed to $\Omega$ itself). This problem was investigated in [44]. We believe that the results presented here can be applied to the subdomain case when MINRES is employed with a block-diagonal preconditioner.

Another class of methods that has proven to be of interest is based on the fact that for some preconditioners the preconditioned saddle point matrix $\mathcal{P}^{-1} \mathcal{K}$ is symmetric and positive definite in an inner product defined by a matrix $\mathcal{H}$, i.e., $\left\langle\mathcal{P}^{-1} A x, y\right\rangle_{\mathcal{H}}=\left\langle x, \mathcal{P}^{-1} A y\right\rangle_{\mathcal{H}}$ where $\langle x, y\rangle_{\mathcal{H}}=x^{T} \mathcal{H} y$. There exists a variety of such methods $[7,40,16,3,29,10]$, which can also be combined to give rise to new methods [43, 41]. Herzog and Sachs [23] analyzed the method of Schöberl and Zulehner [40] for state and control constrained optimal control problems.

We wish to focus our attention on the so-called Bramble-Pasciak CG method introduced in [7], a method that uses a block-triangular preconditioner

$$
\mathcal{P}=\left[\begin{array}{ccc}
A_{0} & 0 & 0 \\
0 & A_{1} & 0 \\
-K & M & -\widehat{S}
\end{array}\right]
$$

with $A_{0}, A_{1}$, and $\widehat{S}$ being approximations just as above. Once the preconditioner is applied to $\mathcal{K}$, the resulting preconditioned matrix $\widehat{\mathcal{K}}=\mathcal{P}^{-1} \mathcal{K}$ is not symmetric anymore but self adjoint in a nonstandard inner product defined by

$$
\mathcal{H}=\left[\begin{array}{ccc}
L-A_{0} & 0 & 0 \\
0 & \beta M-A_{1} & 0 \\
0 & 0 & \widehat{S}
\end{array}\right] .
$$

It is clear that for $\mathcal{H}$ to define an inner product the diagonal blocks have to be symmetric and positive definite. While this is in general a rather tricky issue requiring an eigenvalue estimation problem, in the case of (lumped) mass matrices scaling is straightforward [46]. Further, for the case of a consistent mass matrix, Rees and Stoll showed that the scaling issues can be easily removed [36]. For more details on the implementation and properties of the non-standard inner product solver we refer to $[7,12,23,41,37,36,40]$ and Algorithm 1 below. 


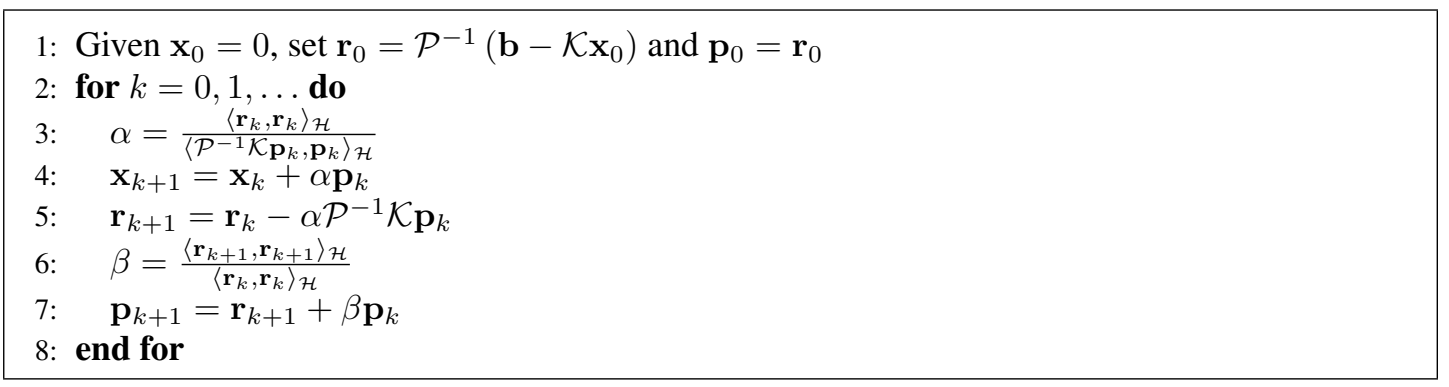

Algorithm 1: Non-standard inner product CG

\subsection{First preconditioner}

The Schur complement of $\mathcal{K}$ is given by

$$
S=K L^{-1} K^{T}+\beta^{-1} M .
$$

For the case $L=M$ it was proposed [35] to neglect the term $\beta^{-1} M$, which would in our case result in an approximation $\widehat{S}_{0}=K L^{-1} K^{T}$ to $S$. For a symmetric system the clustering of the eigenvalues will govern the convergence of the iterative scheme and we want to analyze the eigenvalue distribution of $\widehat{\mathcal{K}}=\mathcal{P}^{-1} \mathcal{K}$ for an idealized case. We consider now the block-triangular preconditioner with the choice $A_{0}=L, A_{1}=\beta M$ and $\widehat{S}_{0}=K L^{-1} K^{T}$, then the eigenvalues of the preconditioned matrix $\mathcal{P}^{-1} \mathcal{K}$ can be read off the diagonal blocks, i.e.,

$$
\mathcal{P}^{-1} \mathcal{K}=\left[\begin{array}{ccc}
I & 0 & -L^{-1} K^{T} \\
0 & I & \beta^{-1} I \\
0 & 0 & I+\beta^{-1} K^{-T} L K^{-1} M
\end{array}\right]
$$

which shows that there are $2 n$ eigenvalues equal to 1 , and $n$ eigenvalues are given by the eigenvalues of $I+\beta^{-1} K^{-T} L K^{-1} M$. Thus, we wish to find eigenvalue bounds for $I+\beta^{-1} K^{-T} L K^{-1} M$. The eigenvalue bounds may be obtained from a field of value analysis ${ }^{\dagger}$. Note that the matrix $I+\beta^{-1} K^{-T} L K^{-1} M$ is similar to the symmetric matrix $M^{1 / 2}\left(I+\beta^{-1} K^{-T} L K^{-1} M\right) M^{-1 / 2}=$ $I+\beta^{-1} M^{1 / 2} K^{-T} L K^{-1} M^{1 / 2}$ and

$$
\frac{x^{T} x+\beta^{-1} x^{T} M^{1 / 2} K^{-T} L K^{-1} M^{1 / 2} x}{x^{T} x}=1+\frac{\beta^{-1}\left(z^{T} L z\right)\left(x^{T} M x\right)\left(y^{T} K^{-T} K^{-1} y\right)}{\left(x^{T} x\right)\left(y^{T} y\right)\left(z^{T} z\right)}
$$

with $y=M^{1 / 2} x$ and $z=K^{-1} y$. The second term on the right hand side of (19) can be bounded using the results of Proposition 1.29 and Theorem 1.32 in [13], which provide bounds for the eigenvalues of the consistent mass matrix and the stiffness matrix. Namely, with $h$ being the meshsize of our finite element we have that

$$
c h^{2} \leq \frac{x^{T} M x}{x^{T} x} \leq C h^{2} \text { and } d h^{2} \leq \frac{x^{T} K x}{x^{T} x} \leq D
$$

with $c, C, d$, and $D$ being mesh-independent constants. Note that these are the bounds for a twodimensional problem. For three-dimensional bounds we also refer to [13] but do not discuss them here. This directly gives bounds for almost all the terms in (19) and the only term that we need to analyze further is $z^{T} L z / z^{T} z$. Using the definition of $L$ we get $\left(z^{T} M z+\varepsilon^{-1} z^{T} G_{\mathcal{A}} M G_{\mathcal{A}} z\right) / z^{T} z$, which obviously can be bounded above by $\left(1+\varepsilon^{-1}\right) C h^{2}$. Hence, the overall bound is given by

$$
\lambda_{\max }^{\left(I+\beta^{-1} K^{-T} L K^{-1} M\right)} \leq 1+\frac{C^{2}}{\beta d^{2}}+\frac{C^{2}}{\beta \varepsilon d^{2}} .
$$

\footnotetext{
${ }^{\dagger}$ The field of values of a matrix $A \in \mathbb{R}^{n, n}$ is a set given by $\frac{x^{T} A x}{x^{T} x} \forall x \neq 0, x \in \mathbb{R}^{n}$.
} 
Similarly the minimum eigenvalues are given by

$$
1 \leq 1+\frac{c^{2} h^{4}}{\beta D^{2}}+\frac{c^{2} h^{4}}{\beta \varepsilon D^{2}} \leq \lambda_{\min }^{\left(I+\beta^{-1} K^{-T} L K^{-1} M\right)} .
$$

Theorem 3.1. For the consistent mass matrix $M$ and the stiffness matrix $K$ of a $Q 1$ finite element space, the eigenvalues of the matrix

$$
I+\beta^{-1} K^{-T} L K^{-1} M
$$

lie in the interval $\left[1,1+\frac{C^{2}}{\beta d^{2}}+\frac{C^{2}}{\beta \varepsilon d^{2}}\right]$.

We remark that the eigenvalue distribution depends on the regularization parameter $\beta$ as was previously observed for other cases (see [35, 46]). It also depends on the values of the penalty parameter $\varepsilon$ : with decreasing value of $\varepsilon$ the upper bound for the eigenvalues in Theorem 20 will increase.

We used the block $\widehat{S}_{0}=K M^{-1} K$ as an approximation for the Schur complement of the system matrix $\mathcal{K}$. This choice results in good clustering of the eigenvalues, but is too expensive for practical purposes as $\widehat{S}_{0}^{-1}$ involves the term $K^{-1}$ (the discretized PDE) twice. One now has to approximate the matrix $K$ as best as possible. For this it is very important to take the structure of the infinite-dimensional problem into account. For both PDEs (2) and (3) the underlying operators are elliptic PDEs and hence multigrid provides a suitable and optimal preconditioner. The most efficient method would certainly be a geometric multigrid method as described in [21, 50]. It is well known that algebraic multigrid method provide very good approximations to the above operators while allowing greater flexibility than their geometric counterparts [38, 14]. As we implemented our method within the deal.II framework [1] we use the available interface to Trilinos [22] and the smoothed aggregation AMG implemented there [17]. Our choice will be to approximate $K$ by a small number of $\mathrm{V}$-cycles and a fixed number of steps of a Chebyshev smoother. The mass matrix $M$ can be efficiently approximated using a variety of methods. In our case, as we only work with lumped mass matrices, we can effortlessly solve for $M$. For consistent mass matrices the Chebyshev semi-iteration $[18,19]$ provides a powerful preconditioner $[49,36]$.

\subsection{Two improved preconditioners}

As we have seen in Theorem 3.1 the regularization parameters $\beta$ and $\varepsilon$ enter into the eigenvalue bounds of the preconditioned matrix. Based on recent efforts [34, 33, 47] we wish to reduce the dependence on the regularization parameters within the preconditioned matrix. For this we motivate a new preconditioner. It is based on the observation that all the mass matrices are lumped and hence the matrix $L$ can be split up in the following way

$$
L=\left[\begin{array}{cc}
M_{\mathcal{I}} & 0 \\
0 & \left(1+\varepsilon^{-1}\right) M_{\mathcal{A}}
\end{array}\right]
$$

where $M_{\mathcal{I}}$ is the part of the mass matrix that corresponds to the free variables and $M_{\mathcal{A}}$ analogously to the active sets. Our aim is to propose a preconditioner of the form

$$
\widehat{S}_{1}=(K+\widehat{M}) L^{-1}(K+\widehat{M})
$$

where $\widehat{S}_{1}$ now approximates the Schur complement $S=K L^{-1} K+\beta^{-1} M$ better than $\widehat{S}_{0}$. Hence, we examine $\widehat{S}_{1}$ in more detail

$$
\widehat{S}_{1}=K L^{-1} K+\widehat{M} L^{-1} \widehat{M}+K L^{-1} \widehat{M}+\widehat{M} L^{-1} K,
$$

and look for a way for $\widehat{M} L^{-1} \widehat{M}$ to approximate the term $\beta^{-1} M$ in the best possible manner. Writing

$$
\widehat{M}=\left[\begin{array}{cc}
\alpha M_{\mathcal{I}} & 0 \\
0 & \gamma M_{\mathcal{A}}
\end{array}\right]
$$


for some parameters $\alpha$ and $\gamma$, gives that if $\widehat{M} L^{-1} \widehat{M}=\beta^{-1} M$, then

$$
\left[\begin{array}{cc}
\alpha^{2} M_{\mathcal{I}} & 0 \\
0 & \gamma^{2}\left(1+\varepsilon^{-1}\right)^{-1} M_{\mathcal{A}}
\end{array}\right]=\widehat{M} L^{-1} \widehat{M}=\beta^{-1} M=\left[\begin{array}{cc}
\beta^{-1} M_{\mathcal{I}} & 0 \\
0 & \beta^{-1} M_{\mathcal{A}}
\end{array}\right] .
$$

This yields that

$$
\alpha=\frac{1}{\sqrt{\beta}} \text { and } \gamma=\frac{\sqrt{1+\varepsilon^{-1}}}{\sqrt{\beta}},
$$

which we then use for $\widehat{M}$ in our approximation to $\widehat{S}_{1}$ in (22). The question now is whether this results in better bounds for the eigenvalues of the preconditioned matrix. For this we are interested in the eigenvalues of the matrix $\widehat{S}_{1}^{-1} S-$ a bound for the eigenvalues of this matrix in the 2D case is presented in Lemma 3.1 below. The 3D case is similar.

Lemma 3.1. For values $\chi \in\left[d h^{2}, D\right]$, which correspond to eigenvalues of $K$, the eigenvalues $\lambda$ of $\widehat{S}_{1}^{-1} S$ satisfy

$$
\lambda\left(\widehat{S}_{1}^{-1} S\right) \in\left[\frac{1}{2},\left(1-\frac{2}{\omega+\omega^{-1}}\right)^{-1}\right],
$$

where $\omega=\beta^{1 / 2} \chi \eta^{-1 / 2} h^{-2}>0$ up to a multiplicative constant of $\mathcal{O}(1)$, with $\eta$ equal to either 1 or $1+\epsilon^{-1}$.

Proof. The eigenvalues of the matrix $\widehat{S}_{1}^{-1} S$ are bounded by the extreme values of the Rayleigh quotient

$$
\begin{aligned}
\frac{v^{T} S v}{v^{T} \widehat{S}_{1} v} & =\frac{v^{T} K L^{-1} K v+\beta^{-1} v^{T} M v}{v^{T} K L^{-1} K v+\beta^{-1} v^{T} M v+v^{T} \widehat{M} L^{-1} K v+v^{T} K L^{-1} \widehat{M} v} \\
& =\left(1+\frac{v^{T} \widehat{M} L^{-1} K v+v^{T} K L^{-1} \widehat{M} v}{v^{T} K L^{-1} K v+\beta^{-1} v^{T} M v}\right)^{-1}=: R .
\end{aligned}
$$

The term of interest here is

$$
\frac{v^{T} \widehat{M} L^{-1} K v+v^{T} K L^{-1} \widehat{M} v}{v^{T} K L^{-1} K v+\beta^{-1} v^{T} M v}=\frac{b^{T} a+a^{T} b}{a^{T} a+b^{T} b}
$$

with $a=L^{-1 / 2} K v$ and $b=L^{-1 / 2} \widehat{M} v$. We note first that we may write

$$
(a-b)^{T}(a-b) \geq 0 \Leftrightarrow \frac{a^{T} b+b^{T} a}{a^{T} a+b^{T} b} \leq 1
$$

for any $a, b$. Using this along with the fact that $a^{T} a+b^{T} b>0$ gives immediately that $R \geq \frac{1}{2}$ for all $v$.

For the upper bound of $R$, we first note that, excluding multiplicative constants of $\mathcal{O}(1), \quad \lambda(K) \in\left[h^{2}, 1\right], \quad \lambda(M)=h^{2}, \quad \lambda(L)=\left\{1,1+\epsilon^{-1}\right\} . \quad$ Further, $\quad$ as $\quad \widehat{M} L^{-1}=L^{-1} \widehat{M}=$ $\frac{1}{\sqrt{\beta}}\left[\begin{array}{cc}I & 0 \\ 0 & \left(1+\epsilon^{-1}\right)^{-1 / 2} I\end{array}\right]$, we have that $\lambda\left(\widehat{M} L^{-1}\right)=\lambda\left(L^{-1} \widehat{M}\right) \in\left\{\beta^{-1 / 2}, \beta^{-1 / 2}(1+\right.$ $\left.\left.\epsilon^{-1}\right)^{-1 / 2}\right\}$. We are now in a position to consider the upper bound of $R$, which corresponds to the largest negative value of $\frac{b^{T} a+a^{T} b}{a^{T} a+b^{T} b}$. We write (using the eigenvalue bounds stated)

$$
\frac{b^{T} a+a^{T} b}{a^{T} a+b^{T} b} \geq-\frac{2 \beta^{-1 / 2} \chi \eta^{-1 / 2}}{\chi^{2} \eta^{-1} h^{-2}+\beta^{-1} h^{2}}=-\frac{2}{\omega+\omega^{-1}}, \quad \text { where } \omega=\beta^{1 / 2} \chi \eta^{-1 / 2} h^{-2}>0,
$$

again excluding multiplicative constants. Therefore

$$
R \leq\left(1-\frac{2}{\omega+\omega^{-1}}\right)^{-1},
$$


and the result is proved.

We observe that we cannot prove a universal, clean upper bound for $\lambda\left(\widehat{S}_{1}^{-1} S\right)$, due to the nonsymmetry of the matrices $\widehat{M} L^{-1} K$ and its transpose. However, we note that if $\omega$ is not too close to 1 , the upper bound of Lemma 3.1 will be close to 1 . We observe that in many practical cases, it is the case that $\omega$ is much smaller than 1 , and therefore our Schur complement approximation is robust in these cases (as well as those where $\omega$ is much greater than 1) ${ }^{\ddagger}$ In Figure 1, we present graphs of eigenvalues of $\widehat{S}_{1}^{-1} S$ for a variety of values of $h, \beta$ and $\epsilon$ to demonstrate that in almost all cases of practical interest, our Schur complement approximation is highly effective. The figures consist of parameter regimes which are close to the worst case in terms of the largest eigenvalue of $\widehat{S}_{1}^{-1} S$.

(a) Different cardinalities of the active set for $\beta=$

$$
1 e-2 \text { and } \varepsilon=1 e-6 \text {. }
$$

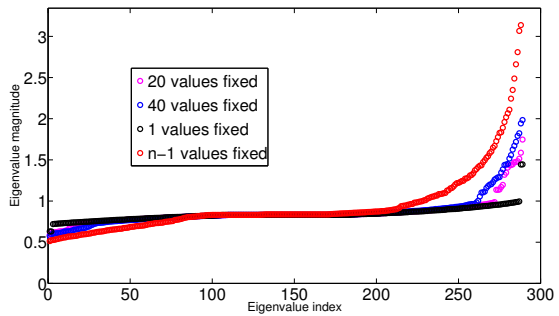

(c) For $n-1$ degrees of freedom in the active sets, varying regularization and penalty parameter.

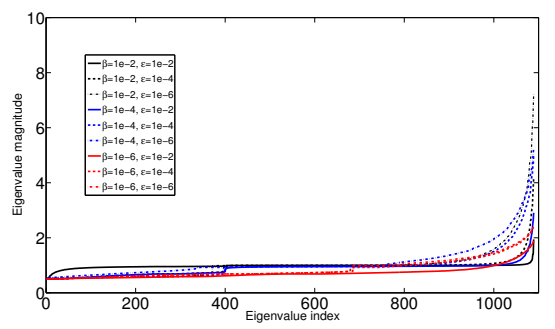

(b) Only one free variable, $\beta=1 e-2$, and $\varepsilon=1 e-$ 6 for varying matrix dimensions.

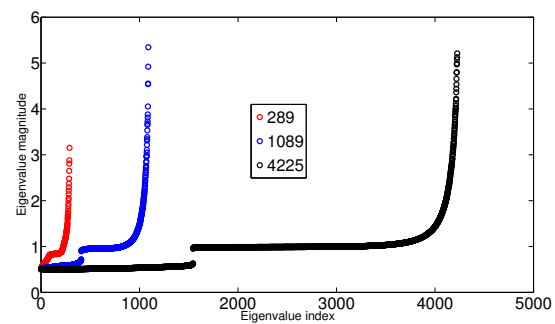

(d) For $n / 2$ degrees of freedom in the active sets and varying regularization parameter.

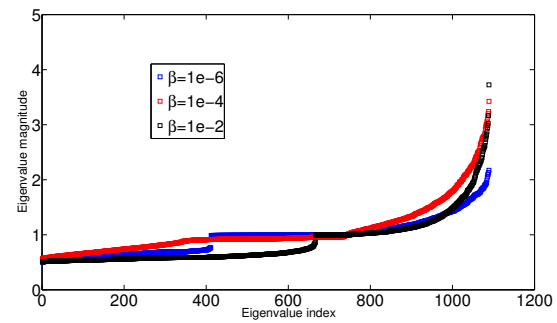

Figure 1. Eigenvalues of the preconditioned Schur-complement.

Another potentially potent Schur complement approximation is identified in the following lemma.

Lemma 3.2. If we approximate the Schur complement $S$ as follows:

$$
\widehat{S}_{2}=\left[K+\frac{1}{\sqrt{\beta}} M\left(I+\frac{1}{\sqrt{\epsilon}} G_{\mathcal{A}}\right)\right] \mathcal{M}_{G}^{-1}\left[K+\frac{1}{\sqrt{\beta}} M\left(I+\frac{1}{\sqrt{\epsilon}} G_{\mathcal{A}}\right)\right]^{T},
$$

where $\mathcal{M}_{G}=\left(I+\frac{1}{\sqrt{\epsilon}} G_{\mathcal{A}}\right) M\left(I+\frac{1}{\sqrt{\epsilon}} G_{\mathcal{A}}\right)$, then the eigenvalues of $\widehat{S}_{2}^{-1} S$ satisfy

$$
\lambda\left(\widehat{S}_{1}^{-1} S\right) \in\left[\frac{1}{2}, 2\left(1-\frac{2}{\nu+\nu^{-1}}\right)^{-1}\right]
$$

where $\nu=\beta^{1 / 2} \chi \zeta^{-1} h^{-2}>0$ up to a multiplicative constant of $\mathcal{O}(1)$. Here, $\chi$ is as defined in Lemma 3.1, and $\zeta$ is either 1 or $1+\epsilon^{-1 / 2}$.

\footnotetext{
‡We note that in the worst case, when $\omega$ is equal to 1 , making a slight adjustment to $\beta$ (say multiplying or dividing it by 4) will ensure that $\omega$ is moved away from 1 , therefore guaranteeing good clustering of the eigenvalues without altering the formulation of the problem in a major way.
} 
Proof. We seek to prove that

$$
S \approx K\left[\left(I+\frac{1}{\sqrt{\epsilon}} G_{\mathcal{A}}\right) M\left(I+\frac{1}{\sqrt{\epsilon}} G_{\mathcal{A}}\right)\right]^{-1} K^{T}+\frac{1}{\beta} M:=\widetilde{S} \approx \widehat{S}_{2},
$$

which we do by considering the eigenvalues of $\widetilde{S}^{-1} S$ and $\widehat{S}_{2}^{-1} \widetilde{S}$, using Rayleigh quotients.

We first examine the Rayleigh quotient

$$
R_{1}:=\frac{v^{T}\left(M+\epsilon^{-1} G_{\mathcal{A}} M G_{\mathcal{A}}\right) v}{v^{T}\left(I+\epsilon^{-1 / 2} G_{\mathcal{A}}\right) M\left(I+\epsilon^{-1 / 2} G_{\mathcal{A}}\right) v}=\frac{a_{1}^{T} a_{1}+b_{1}^{T} b_{1}}{\left(a_{1}+b_{1}\right)^{T}\left(a_{1}+b_{1}\right)},
$$

where $a_{1}=M^{1 / 2} v$ and $b_{1}=\epsilon^{-1 / 2} M^{1 / 2} G_{\mathcal{A}} v$. We can see by straightforward algebra (using that $a_{1}^{T} a_{1}>0$ by positive definiteness of $M$ ) that $R_{1} \geq \frac{1}{2}$. Further, using the fact that $a_{1}^{T} b_{1}=b_{1}^{T} a_{1} \geq 0$ (by virtue of the diagonal and positive definite structure of the lumped mass matrix $M$, and the diagonal and positive semi-definite structure of the projection matrix $G_{\mathcal{A}}$ ), we can show that $R_{1} \leq 1$. From these bounds, it is a simple matter to show that the Rayleigh quotient $\frac{v^{T} S v}{v^{T} \widetilde{S} v} \in[1,2]$.

Looking now at the eigenvalues of $\widehat{S}_{2}^{-1} \widetilde{S}$, we examine the Rayleigh quotient $\frac{v^{T} \widetilde{S} v}{v^{T} \widehat{S}_{2} v}$, writing

$$
R_{2}:=\frac{v^{T} \widetilde{S} v}{v^{T} \widehat{S}_{2} v}=\frac{a_{2}^{T} a_{2}+b_{2}^{T} b_{2}}{\left(a_{2}+b_{2}\right)^{T}\left(a_{2}+b_{2}\right)}=\left(1+\frac{a_{2}^{T} b_{2}+b_{2}^{T} a_{2}}{a_{2}^{T} a_{2}+b_{2}^{T} b_{2}}\right)^{-1},
$$

where $a_{2}=M^{-1 / 2}\left(I+\epsilon^{-1 / 2} G_{\mathcal{A}}\right)^{-1} K^{T} v$ and $b_{2}=\beta^{-1 / 2} M^{1 / 2} v$. By algebraic manipulation (using that $b_{2}^{T} b_{2}>0$ ), it is clear that $\frac{a_{2}^{T} b_{2}+b_{2}^{T} a_{2}}{a_{2}^{T} a_{2}+b_{2}^{T} b_{2}} \leq 1$, and hence that $R_{2} \geq \frac{1}{2}$. For the upper bound of $R_{2}$, we consider the maximum negative value of $\frac{a_{2}^{T} b_{2}+b_{2}^{T} a_{2}}{a_{2}^{T} a_{2}+b_{2}^{T} b_{2}}$. Using that (excluding multiplicative constants of $\mathcal{O}(1)) \lambda(K) \in\left[h^{2}, 1\right], \lambda(M)=h^{2}$ and $\lambda\left(I+\epsilon^{-1 / 2} G_{\mathcal{A}}\right) \in\left\{1,1+\epsilon^{-1 / 2}\right\}$, we may use a very similar approach as in Lemma 3.1 to write that

$$
\frac{a_{2}^{T} b_{2}+b_{2}^{T} a_{2}}{a_{2}^{T} a_{2}+b_{2}^{T} b_{2}} \geq-\frac{2 \beta^{-1 / 2} \chi \zeta^{-1}}{\chi^{2} \zeta^{-2} h^{-2}+\beta^{-1} h^{2}}=-\frac{2}{\nu+\nu^{-1}}, \quad \text { where } \nu=\beta^{1 / 2} \chi \zeta^{-1} h^{-2}>0,
$$

and therefore that $R_{2} \leq\left(1-\frac{2}{\nu+\nu^{-1}}\right)^{-1}$.

Finally, using the above results, we have that the Rayleigh quotient

$$
\frac{v^{T} S v}{v^{T} \widehat{S}_{2} v}=\frac{v^{T} S v}{v^{T} \widetilde{S} v} \cdot \frac{v^{T} \widetilde{S} v}{v^{T} \widehat{S}_{2} v} \in\left[\frac{1}{2}, 2\left(1-\frac{2}{\nu+\nu^{-1}}\right)^{-1}\right],
$$

and so the result is proved.

It is clear that the bound proved in Lemma 3.2 is tight if $\nu$ is far from 1. We again find that this is frequently the case in practical situations, and provide numerical evidence to demonstrate that the eigenvalue bound proved in Lemma 3.2 is indeed a useful one in the vast majority of such cases. Figure 2 shows the eigenvalues for a small example using the Schur complement approximation $\widehat{S}_{2}$. We believe that both $\widehat{S}_{1}$ and $\widehat{S}_{2}$ are viable and effective Schur complement approximations for the problem we are considering.

As we only focus on lumped mass matrices in this paper we refrain from showing results for the non-lumped case in Section 4, though some results still hold for consistent mass matrices.

We note that the analytical results of this section were obtained for an idealized case where we use approximations of the form

$$
\widehat{S}=(K+\widehat{M}) L^{-1}(K+\widehat{M}) \text { with } \widehat{S}^{-1}=(K+\widehat{M})^{-1} L(K+\widehat{M})^{-1} .
$$

However in practice we always use

$$
\widehat{S}^{-1}=\widehat{(\widehat{K+\widehat{M}}}^{-1} L\left(\widehat{K+\widehat{M}}^{-1},\right.
$$




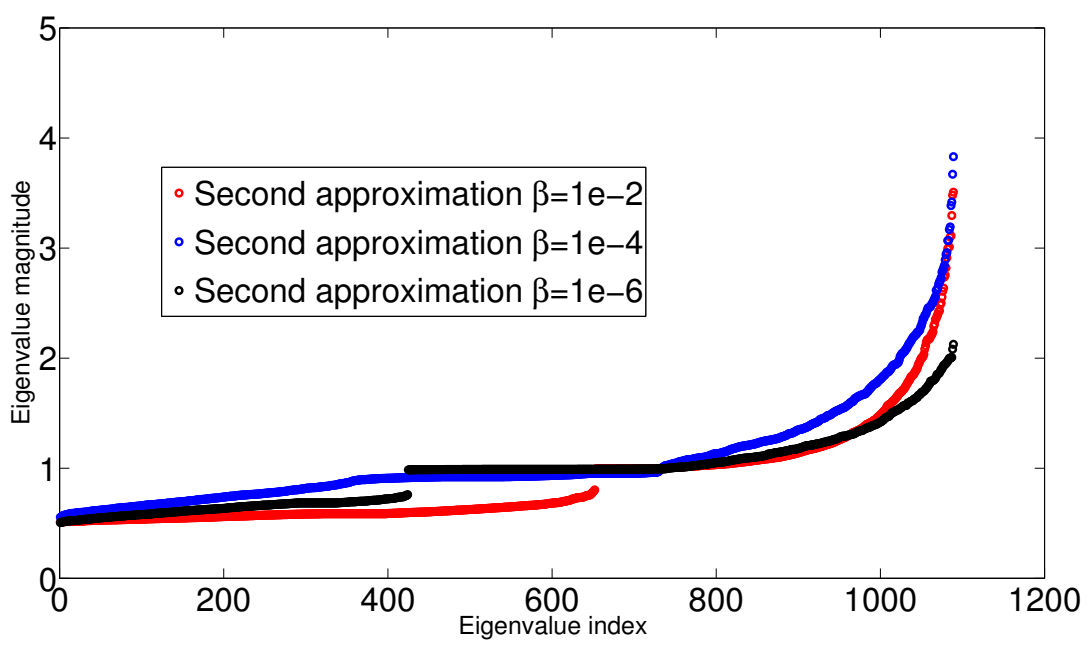

Figure 2. Eigenvalues for $n / 2$ variables in the active sets and varying values of $\beta$

where

$$
\widehat{(K+\widehat{M})}^{-1}
$$

denotes the application of an algebraic or geometric multigrid method to the matrix $K+\widehat{M}$. Note that as $\widehat{M}$ changes with every Newton iteration, we must recompute it at the beginning of each Newton step. Nevertheless, the reduction in iteration numbers is so significant that this is clearly the preferred approach, especially for small values of the regularization parameters.

\subsection{Nested approach}

A strategy that will prove useful in the context of solving state constrained problems is the socalled nested approach [23]. This technique starts by computing the solution to the state constrained problem on a very coarse grid. In the next step a uniform refinement is performed for the mesh, and the solution from the coarse level is prolonged onto the fine mesh. This solution is then used as an initial guess for the Newton method on the fine level. Once the solution is computed to a desired accuracy we can proceed in the same way onto the next finer grid. It is hoped (and will be shown in the next section) that this strategy reduces the number of Newton steps significantly.

\section{NUMERICAL EXPERIMENTS}

All results shown in this section were computed using the deal.II [1] framework with an implementation of the Bramble-Pasciak CG method that uses the 2-norm of the relative preconditioned residual $\left(10^{-6}\right)$ as the stopping criterion. The Newton method is stopped whenever the active sets stay unchanged [4]. For the approximation via algebraic multigrid (AMG) we use 10 steps of a Chebyshev smoother and $4 \mathrm{~V}$-cycles of the smoothed aggregation algebraic multigrid implemented in Trilinos [17]. As the domain $\Omega$ we consider the unit square or cube. All results are performed on a Centos Linux machine with Intel(R) Xeon(R) CPU X5650 @ 2.67GHz CPUs and $48 \mathrm{~GB}$ of RAM. 


\subsection{Results for Dirichlet problems}

$2 D$ results The first example we compute is a Dirichlet problem with boundary condition $y=$ $P_{[\underline{y}, \bar{y}]}\left(y_{d}\right)$ on $\partial \Omega$ defined by

$$
P_{[\underline{y}, \bar{y}]} y_{i}= \begin{cases}y_{d i} & \text { if } \underline{y}_{i}<y_{i}<\bar{y}_{i} \\ \bar{y}_{i} & \text { if } y_{i} \geq \bar{y}_{i} \\ \underline{y}_{i} & \text { if } y_{i} \leq \underline{y}_{i} .\end{cases}
$$

Figure 3 shows the desired state $y_{d}$, computed control $u$ and state $y$ for the case without bound constraints. In Figure 4 we show the computed state and control for a bound constrained problem. The problem is unconstrained from below and the upper bound is given by $\bar{y}=0.1$. It can be seen that there is a small active set where $y_{d}$ is attained which results in the 'hole' in the control (see Figure $4 \mathrm{~b}$ and the active set (black contour) in $4 \mathrm{a}$ ). Here the desired state is given by

$$
y_{d}=\sin \left(2 \pi x_{1} x_{2}\right) .
$$

Table I shows results for $\beta=1 e-2, \varepsilon=1 e-6$ and the upper bound $\bar{y}=0.1$. It can be seen that for this setup the preconditioner as well as the Newton method are almost independent of the mesh parameter. In our experience the performance of the algebraic multigrid preconditioner deteriorates for meshes with smaller mesh size $h$. The increase in iteration numbers could not be observed if a factorization of $K+\widehat{M}$ was used, which for large problems is not feasible. Hence, we chose a rather large number of $\mathrm{V}$-cycles, namely 4 , to approximate the matrix well. A parameter independent approximation of $K+\widehat{M}$ should be investigated in future research. Note that the timings shown also include the setup of the preconditioner for each Newton step in the improved preconditioner. As we can see from Table II where we show results for the same setup but with the non-robust preconditioner presented in Section 3.1, the improvement is substantial as for the setup with $\beta=1 e-2, \varepsilon=1 e-6$, however the Newton method did not converge within 50 iterations. For $\beta=1 e-2, \varepsilon=1 e-4$ we show the results with 4 multigrid cycles in Table III and observe good convergence for this setup of parameters. As for the non-robust preconditioner, the algebraic multigrid only has to approximate $K$ - a smaller number of V-cycles produces the results shown in Table IV. Note that as observed in [27] the quality of the preconditioner determines the convergence of the semi-smooth Newton method with inexact solves.

\begin{tabular}{|c|c|c|c|c|}
\hline DoF & Newton steps & Total CG & CG per Newton & Time for Newton \\
\hline \hline 1089 & 14 & 259 & 18 & 11.27 \\
4225 & 8 & 138 & 17 & 12.63 \\
16641 & 7 & 108 & 15 & 30.3 \\
66049 & 7 & 118 & 16 & 123.54 \\
263169 & 6 & 103 & 17 & 462.78 \\
1050625 & 7 & 183 & 26 & 3267.28 \\
\hline
\end{tabular}

The next comparison we wish to make is that of the quality of the preconditioner for different values of the parameters. As we mentioned earlier some dependence of the AMG on the parameters could be observed. Hence, our choice is on a factorization of $K+\widehat{M}$ for a smaller mesh with 16641 degrees of freedom. The results shown in Table $\mathrm{V}$ show that having no deterioration in the approximation of $K+\widehat{M}$ results in almost constant low iteration numbers for the CG steps per iteration. In practice one should of course use approximations to $K+\widehat{M}$.

$3 D$ results We now wish to show results for the $3 D$ example with the desired state given by

$$
y_{d}=\sin \left(2 \pi x_{1} x_{2} x_{3}\right)
$$




\begin{tabular}{|c|c|c|c|c|}
\hline DoF & Newton steps & Total CG & CG per Newton & Time for Newton \\
\hline \hline 1089 & 14 & 780 & 55 & 29.31 \\
4225 & 2 & 14 & 7 & 1.25 \\
16641 & 5 & 59 & 11 & 13.63 \\
66049 & 10 & 170 & 17 & 131.98 \\
\hline 263169 & \multicolumn{3}{|c|}{ No convergence after 50 Newton steps } \\
\hline
\end{tabular}

Table II. $2 D$-Results for non-zero Dirichlet boundary, $\beta=1 e-2, \varepsilon=1 e-6$ and $\bar{y}=0.1$.

\begin{tabular}{|c|c|c|c|c|}
\hline DoF & Newton steps & Total CG & CG per Newton & Time for Newton \\
\hline \hline 1089 & 9 & 145 & 16 & 5.55 \\
4225 & 3 & 21 & 7 & 1.87 \\
16641 & 4 & 34 & 8 & 7.75 \\
66049 & 4 & 39 & 9 & 31.26 \\
263169 & 9 & 172 & 19 & 530.38 \\
\hline
\end{tabular}

Table III. $2 D$-Results for non-zero Dirichlet boundary, $\beta=1 e-2, \varepsilon=1 e-4$ and $\bar{y}=0.1$ with 4 V-cycles.

\begin{tabular}{|c|c|c|c|c|}
\hline DoF & Newton steps & Total CG & CG per Newton & Time for Newton \\
\hline \hline 1089 & 9 & 150 & 16 & 5.64 \\
4225 & 3 & 24 & 8 & 1.76 \\
16641 & 7 & 124 & 17 & 22.63 \\
66049 & 8 & 307 & 38 & 191.54 \\
263169 & 10 & 1059 & 105 & 2507.48 \\
\hline
\end{tabular}

Table IV. $2 D$-Results for non-zero Dirichlet boundary, $\beta=1 e-2, \varepsilon=1 e-4$ and $\bar{y}=0.1$ with 2 V-cycles.

\begin{tabular}{|c|c|c|c|}
\hline$\varepsilon \downarrow \beta \rightarrow$ & $1 \mathrm{e}-2$ & $1 \mathrm{e}-4$ & $1 \mathrm{e}-6$ \\
\hline \hline $1 \mathrm{e}-4$ & 19 & 27 & 26 \\
$1 \mathrm{e}-6$ & 26 & 34 & 28 \\
$1 \mathrm{e}-8$ & 32 & 36 & 28 \\
\hline
\end{tabular}

Table V. Number of CG iterations per Newton step for different values of $\beta$ and $\varepsilon$, using a direct factorization of $K+\widehat{M}$. The example was again the $2 D$-results for non-zero Dirichlet boundary and $\bar{y}=0.1$.

and a zero Dirichlet boundary condition. In this case we again consider the upper bound $\bar{y}=0.1$, and the parameters $\beta=1 e-2$ and $\varepsilon=1 e-4$. The results shown in Table VI show that the iteration numbers per Newton step as well as the number of Newton steps stays constant.

\begin{tabular}{|c|c|c|c|c|}
\hline DoF & Newton steps & Total CG & CG per Newton & Time for Newton \\
\hline \hline 729 & 4 & 48 & 12 & 1.05 \\
4913 & 4 & 53 & 13 & 6.17 \\
35937 & 4 & 53 & 13 & 37.78 \\
274625 & 3 & 41 & 13 & 228.05 \\
\hline
\end{tabular}

Table VI. $3 D$-Results for zero Dirichlet boundary and $\bar{y}=0.1$. 
(a) Desired state

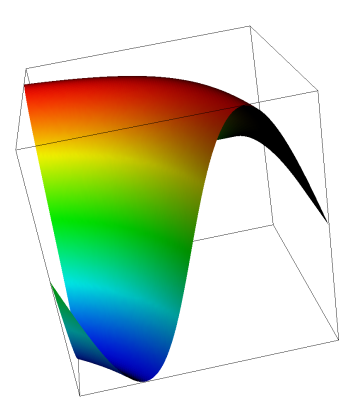

(b) State

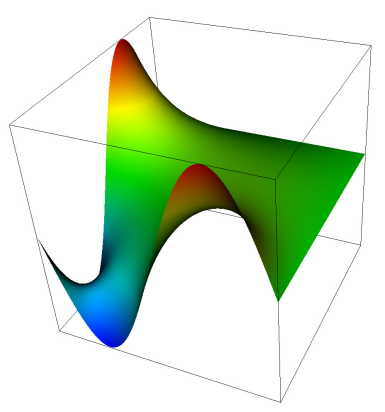

(c) Control

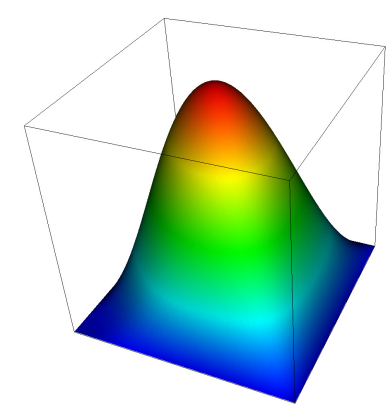

Figure 3. Desired state, state and control for unconstrained problem

(a) Computed state

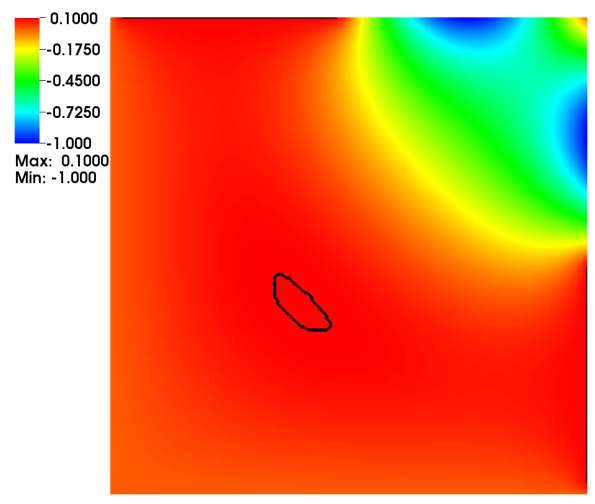

(b) Computed control

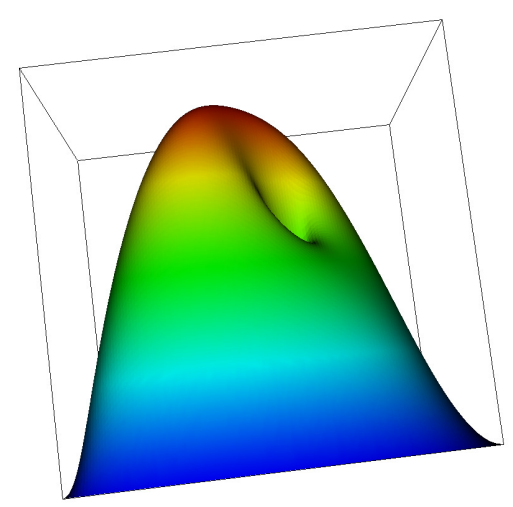

Figure 4. Computed state and control for constrained problem

\subsection{Results for Neumann boundary}

In this section we only consider three-dimensional results for the problem with the state equation given by (3). We start with the desired state given by

$$
y_{d}=\sin \left(2 \pi x_{1} x_{2} x_{3}\right)
$$

and the lower bound $y=0$ - the results are shown in Table VII. Here we take $\beta=1 e-3$ and $\varepsilon=1 e-5$. An illustration of the desired state and the constrained state is shown in Figure 5.

\begin{tabular}{|c|c|c|c|c|}
\hline DoF & Newton steps & Total CG & CG per Newton & Time for Newton \\
\hline \hline 729 & 6 & 78 & 13 & 1.95 \\
4913 & 4 & 63 & 15 & 7.49 \\
35937 & 4 & 72 & 18 & 51.61 \\
274625 & 4 & 75 & 18 & 413.21 \\
2146689 & 5 & 104 & 20 & 4458.58 \\
\hline
\end{tabular}

Table VII. 3D-Results for Neumann boundary and $\underline{y}=0.2$. 
(a) Desired state

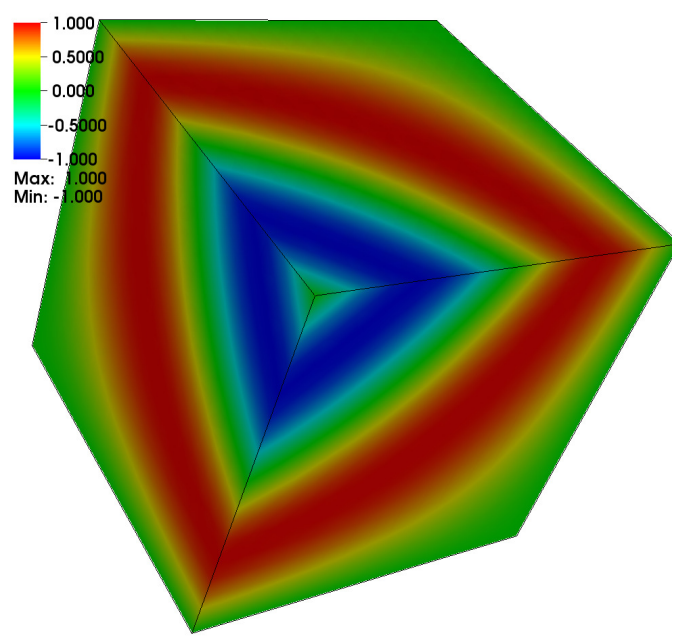

(b) State

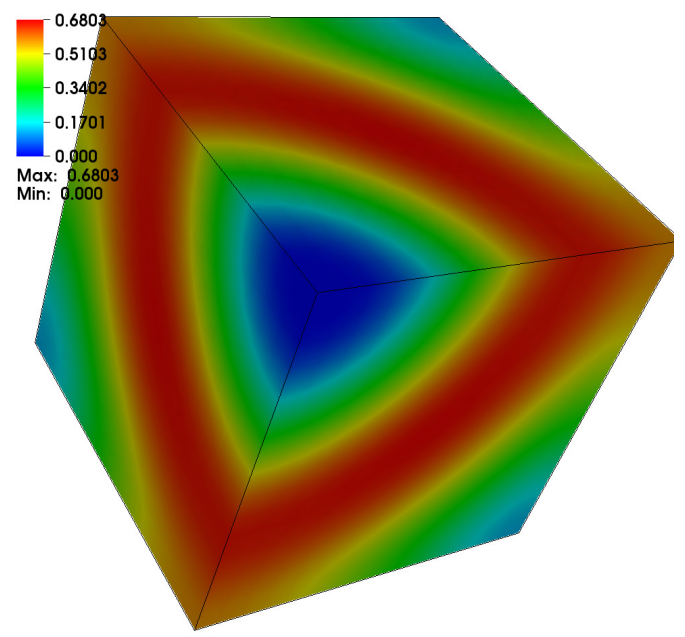

Figure 5. Desired state and state with lower bound $\underline{y}=0$ for problem in $3 D$

We next compute an example presented in [23] where the desired state is given by

$$
y_{d}= \begin{cases}1 & \text { if } x_{0}<0.5 \\ -2 & \text { otherwise }\end{cases}
$$

The upper bound is given by $\bar{y}=0$ and $\beta=1 e-2$ and $\varepsilon=1 e-4$. The results are shown in Table VIII where, in contrast to [23], we observe parameter robust convergence.

\begin{tabular}{|c|c|c|c|c|}
\hline DoF & Newton steps & Total CG & CG per Newton & Time for Newton \\
\hline \hline 729 & 5 & 52 & 10 & 1.32 \\
4913 & 1 & 13 & 13 & 1.67 \\
35937 & 2 & 30 & 15 & 22.19 \\
274625 & 2 & 32 & 16 & 181.56 \\
2146689 & 1 & 16 & 16 & 758.11 \\
\hline
\end{tabular}

Table VIII. $3 D$-Results for Neumann boundary and $\bar{y}$ from Herzog and Sachs.

\section{CONCLUSIONS AND OUTLOOK}

In this paper we introduced preconditioners for a state constrained PDE-constrained optimization problem when solved using the Moreau-Yosida penalization. The Krylov subspace solvers we used showed very promising performance as we could theoretically and practically obtain parameter robust convergence of the preconditioned Krylov solver.

In the future, it would be useful to investigate the problem where the $L^{2}$ norm of $y-y_{d}$ is measured on a subdomain of $\Omega$, as opposed to $\Omega$ itself, as previously discussed. This, like the problem considered in this manuscript, could be solved using the preconditioned MINRES algorithm. Also, the choice of multilevel method for the parameter dependent matrix $K+\widehat{M}$ should be re-considered, as we could observe dependence on the parameters for smaller meshes within the AMG preconditioner. Geometric multigrid and more advanced algebraic multigrid preconditioners 
should be investigated, and incorporation of a more sophisticated scheme for the parameter $\varepsilon$ would also be desirable for future implementations. Finally, a significant piece of future work would be to extend the results presented here to time-dependent problems, as well as to more difficult PDEs.

\section{ACKNOWLEDGMENTS}

The authors would like to thank the anonymous referees for their careful reading of the manuscript and helpful comments. The first author was supported for this work by the Engineering and Physical Sciences Research Council (UK), Grant EP/P505216/1. This publication is partially based on work done when the second author was supported by Award No. KUK-C1-013-04, made by King Abdullah University of Science and Technology (KAUST).

\section{REFERENCES}

1. W. BANGERTH, R. HARTMANN, AND G. KANSCHAT, deal.II-a general-purpose object-oriented finite element library, ACM Transactions on Mathematical Software, 33 (2007), pp. Art. 24, 27.

2. M. Benzi, G. H. Golub, And J. Liesen, Numerical solution of saddle point problems, Acta Numerica, 14 (2005), pp. 1-137.

3. M. BENZI AND V. SimONCINI, On the eigenvalues of a class of saddle point matrices, Numerische Mathematik, 103 (2006), pp. 173-196.

4. M. Bergounioux, K. ITO, AND K. KUnisch, Primal-dual strategy for constrained optimal control problems, SIAM Journal on Control and Optimization , 37 (1999), pp. 1176-1194.

5. M. Bergounioux And K. Kunisch, Primal-dual strategy for state-constrained optimal control problems, Computational Optimization and Applications, 22 (2002), pp. 193-224.

6. L. Blank, L. SARbu, AND M. StOll, Preconditioning for Allen-Cahn variational inequalities with non-local constraints, Submitted, (2011).

7. J. H. BRAMBlE AND J. E. PASCIAK, A preconditioning technique for indefinite systems resulting from mixed approximations of elliptic problems, Mathematics of Computation , 50 (1988), pp. 1-17.

8. E. CASAS, Control of an elliptic problem with pointwise state constraints, SIAM Journal on Control and Optimization, 24 (1986), pp. 1309-1318.

9. T. DAVIS, Umfpack version 4.4 user guide, tech. rep., Dept. of Computer and Information Science and Engineering Univ. of Florida, Gainesville, FL, 2005.

10. C. R. DOHRMANN AND R. B. LeHOUCQ, A primal-based penalty preconditioner for elliptic saddle point systems, SIAM Journal on Numerical Analysis, 44 (2006), pp. 270-282.

11. I. DUfF, Sparse numerical linear algebra: direct methods and preconditioning, Technical Report TR/PA/96/22, CERFACS, Toulouse, France, 1996. Also RAL Report RAL 96-047.

12. H. C. Elman, Multigrid and Krylov subspace methods for the discrete Stokes equations, in Seventh Copper Mountain Conference on Multigrid Methods, N. D. Melson, T. A. Manteuffel, S. F. McCormick, and C. C. Douglas, eds., vol. CP 3339, Hampton, VA, 1996, NASA, pp. 283-299.

13. H. C. ElmAN, D. J. Silvester, AND A. J. WATHEN, Finite elements and fast iterative solvers: with applications in incompressible fluid dynamics, Numerical Mathematics and Scientific Computation, Oxford University Press, New York, 2005.

14. R. Falgout, An Introduction to Algebraic Multigrid, Computing in Science and Engineering, 8 (2006), pp. $24-33$. Special Issue on Multigrid Computing.

15. B. FISCHER, Polynomial based iteration methods for symmetric linear systems, Wiley-Teubner Series Advances in Numerical Mathematics, John Wiley \& Sons Ltd, Chichester, 1996.

16. B. Fischer, A. Ramage, D. J. Silvester, And A. J. WATHEn, Minimum residual methods for augmented systems, BIT, 38 (1998), pp. 527-543.

17. M. Gee, C. Siefert, J. Hu, R. Tuminaro, And M. SAla, ML 5.0 smoothed aggregation user's guide, Tech. Rep. SAND2006-2649, Sandia National Laboratories, 2006.

18. G. H. GOLUB AND R. S. VARGA, Chebyshev semi-iterative methods, successive over-relaxation iterative methods, and second order Richardson iterative methods. I, Numerische Mathematik, 3 (1961), pp. 147-156.

19. _ Chebyshev semi-iterative methods, successive over-relaxation iterative methods, and second order Richardson iterative methods. II, Numerische Mathematik, 3 (1961), pp. 157-168.

20. A. GReEnBAUM, Iterative methods for solving linear systems, vol. 17 of Frontiers in Applied Mathematics, Society for Industrial and Applied Mathematics (SIAM), Philadelphia, PA, 1997.

21. W. HACKBUSCH, Multigrid methods and applications, vol. 4 of Springer Series in Computational Mathematics, Springer-Verlag, Berlin, 1985.

22. M. Heroux, R. Bartlett, V. H. R. Hoekstra, J. Hu, T. Kolda, R. Lehoucq, K. Long, R. Pawlowski, E. Phipps, A. Salinger, H. Thornquist, R. Tuminaro, J. Willenbring, and A. Williams, An Overview of Trilinos, Tech. Rep. SAND2003-2927, Sandia National Laboratories, 2003.

23. R. Herzog AND E. W. SACHS, Preconditioned conjugate gradient method for optimal control problems with control and state constraints, SIAM Journal on Matrix Analysis and Applications, 31 (2010), pp. 2291-2317.

24. M. Hinze, R. Pinnau, M. Ulbrich, And S. Ulbrich, Optimization with PDE Constraints, Mathematical Modelling: Theory and Applications, Springer-Verlag, New York, 2009. 
25. K. ITO AND K. KUNISCH, Semi-smooth Newton methods for state-constrained optimal control problems, Systems Control Lett., 50 (2003), pp. 221-228.

26. - Lagrange multiplier approach to variational problems and applications, vol. 15 of Advances in Design and Control, Society for Industrial and Applied Mathematics (SIAM), Philadelphia, PA, 2008.

27. C. KANZOW, Inexact semismooth newton methods for large-scale complementarity problems, Optimization Methods and Software, 19 (2004), pp. 309-325.

28. K. Krumbiegel, I. Neitzel, AND A. Rösch, Sufficient optimality conditions for the Moreau-Yosida-type regularization concept applied to the semilinear elliptic optmimal control problems with pointwise state constraints, Tech. Rep. 1503/2010, WIAS, 2010.

29. J. LIESEN AND B. N. PARLETT, On nonsymmetric saddle point matrices that allow conjugate gradient iterations, Numerische Mathematik, 108 (2008), pp. 605-624.

30. C. MeYER, U. PRÜFERT, AND F. TRÖLTZSCH, On two numerical methods for state-constrained elliptic control problems, Optimization Methods and Software, 22 (2007), pp. 871-899.

31. M. F. Murphy, G. H. Golub, AND A. J. WATHEN, A note on preconditioning for indefinite linear systems, SIAM Journal on Scientific Computing , 21 (2000), pp. 1969-1972.

32. C. C. PAIGE AND M. A. SAUNDERS, Solutions of sparse indefinite systems of linear equations, SIAM Journal on Numerical Analysis, 12 (1975), pp. 617-629.

33. J. W. PeARSON, M. STOLL, AND A. J. WATHEn, Regularization-robust preconditioners for time-dependent PDEconstrained optimization problems, Submitted, (2011).

34. J. W. Pearson AND A. J. WATHEn, A New Approximation of the Schur Complement in Preconditioners for PDE Constrained Optimization, to appear in Numerical Linear Algebra with Applications, (2010).

35. T. REES, H. S. Dollar, AND A. J. WATHEN, Optimal solvers for PDE-constrained optimization, SIAM Journal on Scientific Computing, 32 (2010), pp. 271-298.

36. T. REES AND M. STOLL, Block-triangular preconditioners for pde-constrained optimization, Numerical Linear Algebra with Applications, 17 (2010), pp. 977-996.

37. T. ReEs, M. Stoll, AND A. WATHEN, All-at-once preconditioners for PDE-constrained optimization, Kybernetika, 46 (2010), pp. 341-360.

38. J. W. RUGE AND K. STÜBEn, Algebraic multigrid, in Multigrid methods, vol. 3 of Frontiers Appl. Math., SIAM, Philadelphia, PA, 1987, pp. 73-130.

39. Y. SAAD, Iterative methods for sparse linear systems, Society for Industrial and Applied Mathematics, Philadelphia, PA, 2003.

40. J. SCHÖBERL AND W. ZULEHNER, Symmetric indefinite preconditioners for saddle point problems with applications to pde-constrained optimization problems, SIAM Journal on Matrix Analysis and Applications, 29 (2007), pp. 752-773.

41. M. STOLL, Solving Linear Systems using the Adjoint, $\mathrm{PhD}$ thesis, University of Oxford, 2009.

42. - All-at-once solution of a time-dependent time-periodic PDE-constrained optimization problems, Submitted, (July 2011).

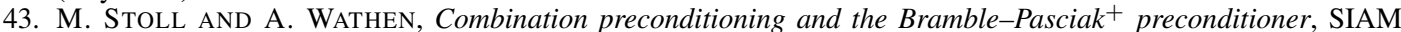
Journal on Matrix Analysis and Applications, 30 (2008), pp. 582-608.

44. M. Stoll AND A. WATHen, All-at-once solution of time-dependent PDE-constrained optimization problems, Submitted, (2010).

45. - All-at-once solution of time-dependent Stokes control, Submitted, (2011).

46. _ Preconditioning for partial differential equation constrained optimization with control constraints, Numerical Linear Algebra with Applications, 19 (2012), pp. 53-71.

47. S. TAKACS AND W. ZULEHNER, Convergence analysis of multigrid methods with collective point smoothers for optimal control problems, Submitted, (2011).

48. F. TRÖLtZSCH, Optimale Steuerung partieller Differentialgleichungen: Theorie, Verfahren und Anwendungen, Vieweg Verlag, Wiesbaden, 2005.

49. A. J. WATHEN AND T. REES, Chebyshev semi-iteration in preconditioning for problems including the mass matrix, Electronic Transactions in Numerical Analysis, 34 (2008), pp. 125-135.

50. P. Wesseling, An introduction to multigrid methods, Pure and Applied Mathematics (New York), John Wiley \& Sons Ltd., Chichester, 1992. 